\title{
Editorial
}

\section{Medicinal Herbs and Their Active Compounds for Fatty Liver Diseases}

\author{
Chang Gue Son, ${ }^{1}$ Zhang Wei, ${ }^{2}$ H. Balaji Raghavendran, ${ }^{3}$ \\ Jing-Hua Wang, ${ }^{4}$ and Elzbieta Janda ${ }^{5}$ \\ ${ }^{1}$ Liver \& Immunology Research Center, Daejeon Oriental Hospital of Daejeon University, Daejeon, Republic of Korea \\ ${ }^{2}$ Shanghai University of Traditional Chinese Medicine, Shanghai, China \\ ${ }^{3}$ Department of Orthopedic Surgery, University of Malaya, Faculty of Medicine, Kuala Lumpur, Malaysia \\ ${ }^{4}$ Key Laboratory of Xinian Medicine, Ministry of Education, Clinical College of TCM, Anhui University of TCM, Hefei, \\ Anhui Province, China \\ ${ }^{5}$ Department of Health Sciences, Magna Graecia University, Campus Germaneto, Catanzaro, Italy \\ Correspondence should be addressed to Chang Gue Son; ckson@dju.ac.kr
}

Received 7 November 2017; Accepted 8 November 2017; Published 7 December 2017

Copyright (c) 2017 Chang Gue Son et al. This is an open access article distributed under the Creative Commons Attribution License, which permits unrestricted use, distribution, and reproduction in any medium, provided the original work is properly cited.

Fatty liver disease (FLD), also called commonly as fatty liver or hepatic steatosis, is a condition of the excessive accumulation of lipids in hepatocytes. The prevalence of FLD in the general population ranges from $10 \%$ to $24 \%$ in various countries [1]. Fatty liver is generally classified as alcoholic steatosis (alcoholic FLD) or nonalcoholic fatty liver disease (NAFLD), depending on the contribution of alcohol to the FLD pathogenesis [2]. Due to decreased number of hepatic viruses carriers and increased population with obesity, FLD has become the most common cause of abnormal liver function tests in developed countries recently [3].

Fatty liver is a leading step to chronic liver diseases including steatohepatitis (nonalcoholic steatohepatitis, NASH, and alcoholic steatohepatitis, ASH), liver fibrosis, and cirrhosis worldwide [4]. In addition, FLD is also associated with other diseases such as metabolic syndrome and diabetes mellitus [5]. FLD has a complex pathology involving the imbalance between lipogenesis and lipolysis, followed by inflammatory response [6]. The high prevalence of FLD is considered as a medical issue worldwide; yet there are no currently available drug-based therapies. For this reason medicinal herbs-derived remedies are emerging as potential therapeutics against FLD due to high efficacy and low risk of side effects $[7,8]$.

This special issue is an attempt to contribute to the knowledge on CAM treatments for fatty liver diseases and its associated disorders. We called for articles that have explored effectiveness and mechanisms of medicinal herbs and their compounds on FLD. A collection of seven original research articles are presented, which address the animal (six articles) or cell-based (one article) pharmacological effects of herbal drugs or their compounds on FLD. This issue presets herbal resources which consisted of three multiherbal formulae (Dahuang Zexie Decoction, Yinchen Linggui Zhugan Decoction, and Seyoeum), three of medicinal plants (Artemisia iwayomogi plus Curcuma longa, Salvia-Nelumbinis Naturalis, and Euphorbia kansui), and one citrus bioflavonoid (Hesperidin) against NAFLD, nonalcoholic steatohepatitis (NASH), and lipid metabolic disorders, respectively.

This special issue provides valuable information to practitioners and researchers working in the field of FLD regarding new potential medicinal herbs and pathophysiological features of multitargets in FLD. We hope that it will become their inspiration or reference to develop new therapeutic strategies against FLD based on herbal-derived multidrugs and bioactive compounds.

\section{Acknowledgments}

We, the editorial team, sincerely thank all the authors for submitting their valuable manuscripts and for their patience, 
and we are also grateful to the reviewers for their timely responses. All the credits for developing this issue go to all its contributors and the guest editors.

\section{Chang Gue Son Zhang Wei \\ H. Balaji Raghavendran Jing-Hua Wang Elzbieta Janda}

\section{References}

[1] W. Dunn, P. Angulo, S. Sanderson et al., "Utility of a new model to diagnose an alcohol basis for steatohepatitis," Gastroenterology, vol. 131, no. 4, pp. 1057-1063, 2006.

[2] P. Angulo, "Medical progress: nonalcoholic fatty liver disease," The New England Journal of Medicine, vol. 346, no. 16, pp. 12211231, 2002.

[3] D. R. LaBrecque, Z. Abbas, F. Anania et al., "World gastroenterology organisation global guidelines: nonalcoholic fatty liver disease and nonalcoholic steatohepatitis," Journal of Clinical Gastroenterology, vol. 48, no. 6, pp. 467-473, 2014.

[4] M. Blachier, H. Leleu, M. Peck-Radosavljevic, D.-C. Valla, and F. Roudot-Thoraval, "The burden of liver disease in Europe: a review of available epidemiological data," Journal of Hepatology, vol. 58, no. 3, pp. 593-608, 2013.

[5] J. C. Bae, S. K. Kim, J. M. Han et al., "Additive effect of nonalcoholic fatty liver disease on the development of diabetes in individuals with metabolic syndrome," Diabetes Research and Clinical Practice, vol. 129, pp. 136-143, 2017.

[6] A. E. Feldstein, N. W. Werneburg, and A. Canbay, "Free fatty acids promote hepatic lipotoxicity by stimulating TNF- $\alpha$ expression via a lysosomal pathway," Hepatology, vol. 40, no. 1 , pp. 185-194, 2004.

[7] M. Parafati, A. Lascala, V. M. Morittu et al., "Bergamot polyphenol fraction prevents nonalcoholic fatty liver disease via stimulation of lipophagy in cafeteria diet-induced rat model of metabolic syndrome," The Journal of Nutritional Biochemistry, vol. 26, no. 9, pp. 938-948, 2015.

[8] H. Yao, Y.-J. Qiao, Y.-L. Zhao et al., "Herbal medicines and nonalcoholic fatty liver disease," World Journal of Gastroenterology, vol. 22, no. 30, pp. 6890-6905, 2016. 


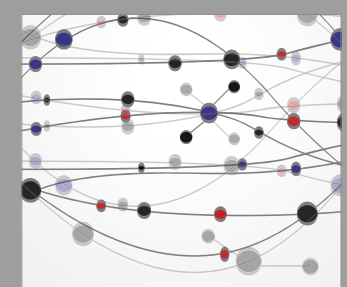

The Scientific World Journal
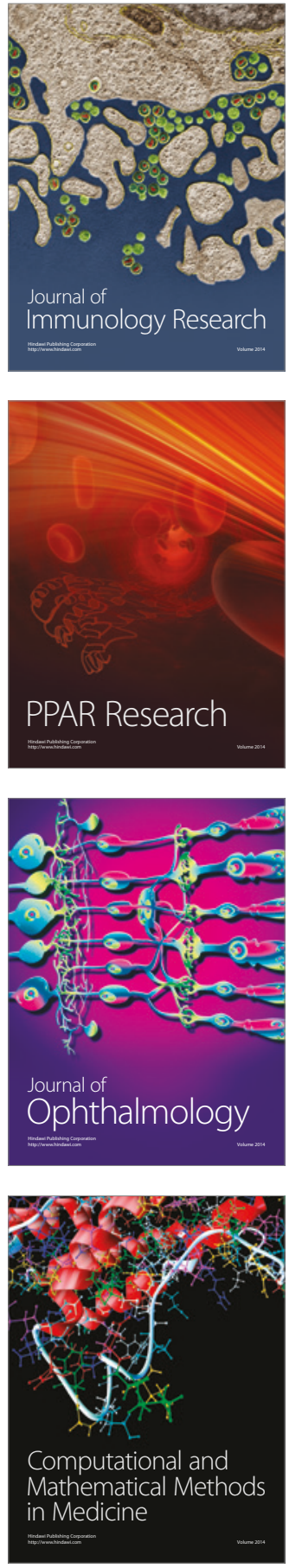

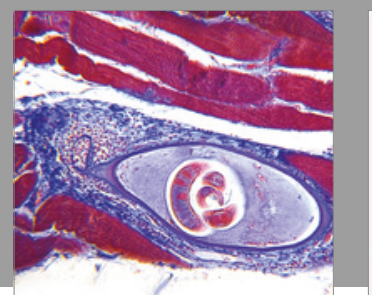

Gastroenterology Research and Practice
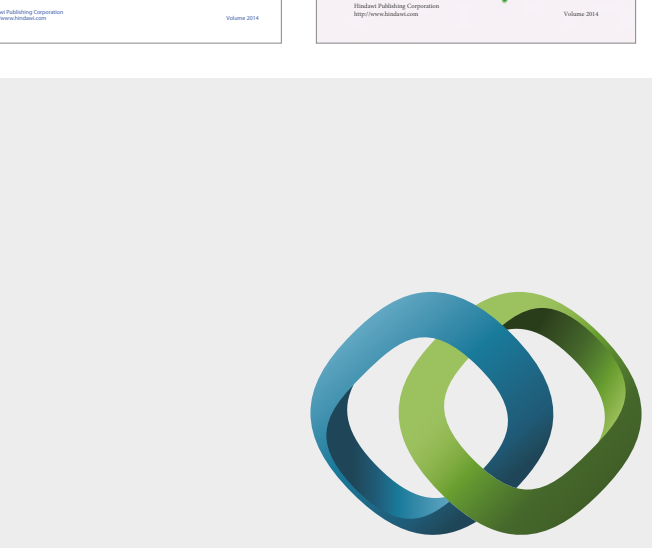

\section{Hindawi}

Submit your manuscripts at

https://www.hindawi.com
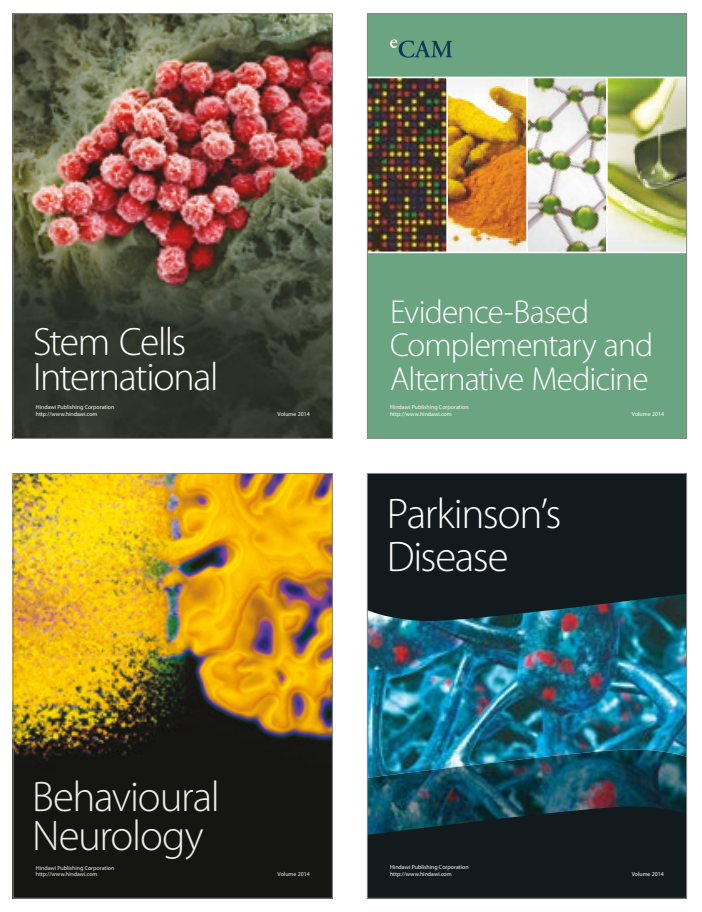
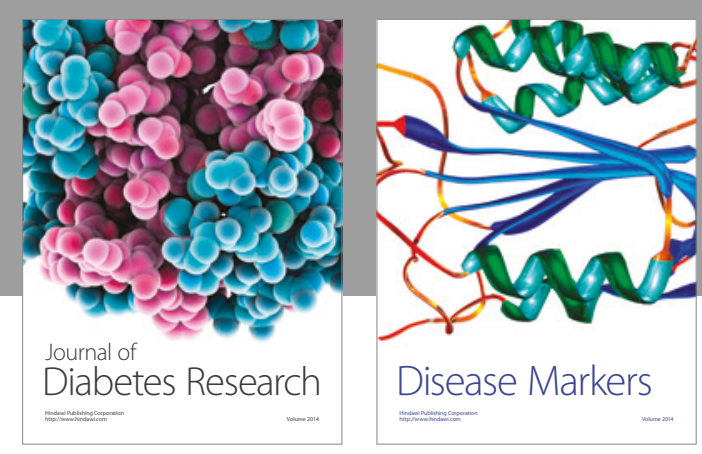

Disease Markers
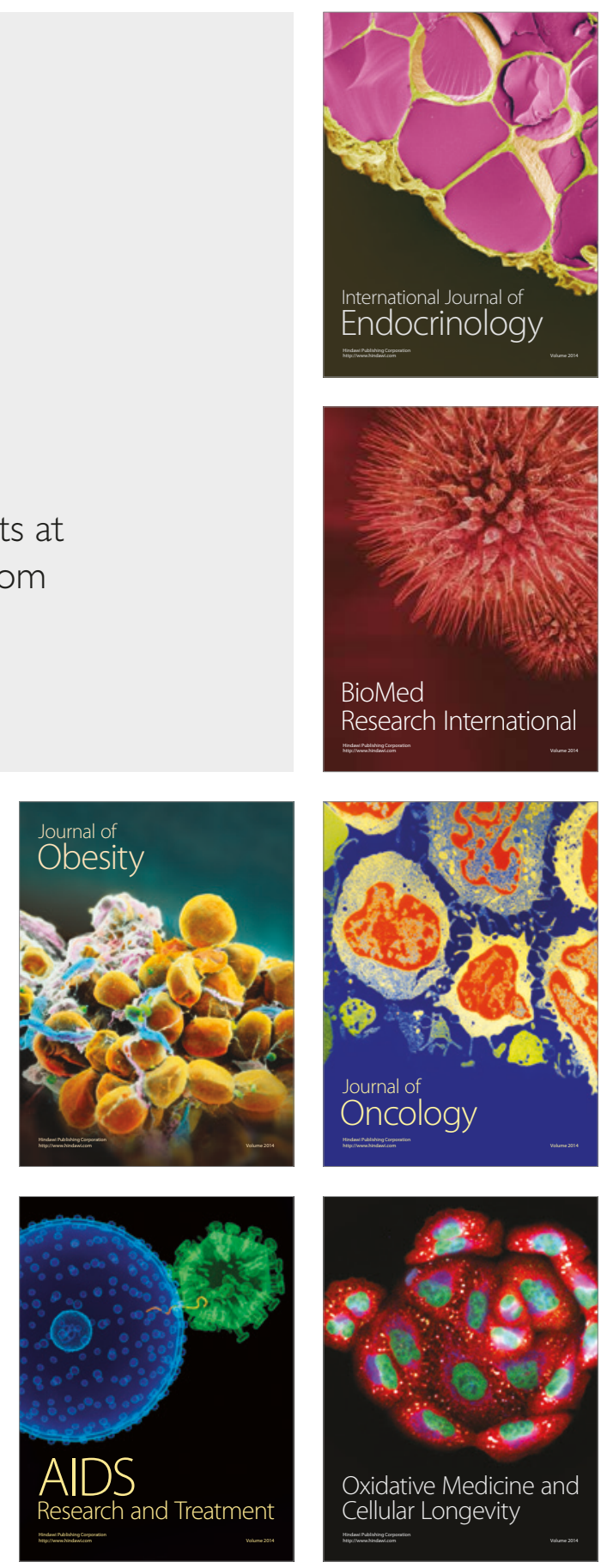